\title{
Evaluation of the hematologic indices in patients with thyrotoxicosis with distinct etiologies: a case-control study
}

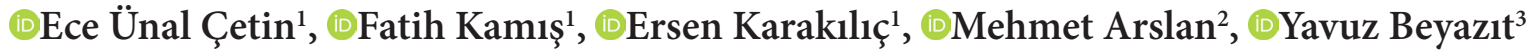 \\ ${ }^{1}$ Çanakkale Onsekiz Mart University School of Medicine, Department of Internal Medicine, Çanakkale, Turkey \\ ${ }^{2}$ Çanakkale Onsekiz Mart University School of Medicine, Department of Cardiology, Çanakkale, Turkey \\ ${ }^{3}$ Çanakkale Onsekiz Mart University School of Medicine, Department of Gastroenterology, Çanakkale, Turkey
}

Cite this article as: Ünal Çetin E, Kamış F, Karakılıç E, Arslan M, Beyazıt Y. Evaluation of the hematologic indices in patients with thyrotoxicosis with distinct etiologies: a case-control study. J Health Sci Med 2021; 4(2): 198-202.

\begin{abstract}
Aim: Thyrotoxicosis is a clinical state of inappropriately high levels of free T4 (thyroxine) and/or free T3 (tri-iodothyronine) in the body caused by distinct etiologies including Graves' disease (GD), subacute thyroiditis (SAT), toxic adenoma and toxic multinodular goiter (TMNG). Simple hematologic indices such as neutrophil/lymphocyte ratio (NLR), platelet/lymphocyte ratio (PLR) and mean platelet volume (MPV) have increasingly been mentioned as measures of presence and severity of thyrotoxicosis. In this study, we aimed to analyze whether there is a link between these peripheral blood parameters and the presence of thyrotoxicosis.

Material and Method: A total of 46 GD, 46 TMNG, 39 TA and 45 SAT patients and 45 healthy controls were included. Laboratory parameters and NLR, PLR, and MPV values were recorded from peripheral blood complete blood cell counts for each patient.

Results: This study showed that NLR and PLR levels are elevated in patients with SAT in comparison with other thyrotoxic patient groups and controls. The post hoc analysis of comparison of NLR and PLR in each study groups revealed that NLR and PLR were statistically different in the SAT group in comparison to the GD, TMNG, TA, and healthy controls. A significant decrease in the level of MPV was demonstrated in thyrotoxicosis patients $(\mathrm{p}<0.001)$.

Conclusion: NLR, PLR and MPV that is routinely and automatically calculated from complete blood count plays an important diagnostic role in thyrotoxicosis.

Keywords: Thyrotoxicosis, mean platelet volume, neutrophil-to-lymphocyte ratio, platelet-to-lymphocyte ratio
\end{abstract}

\section{INTRODUCTION}

Thyrotoxicosis is usually characterized by the clinical manifestations of unsuitably elevated thyroid hormone (TH) action in the body. Hyperthyroidism is a kind of thyrotoxicosis owing to elevated $\mathrm{TH}$ synthesis and secretion in the thyroid gland. Although Graves' disease (GD) is one of the most common reason of hyperthyroidism in iodine-sufficient regions all over the world that affects $<1.0 \%$ of the entire population, the other three most frequent causes of hyperthyroidism are toxic multinodular goiter (TMNG), toxic adenoma (TA), and subacute thyroiditis (SAT) (1-3). Patient characteristics and laboratory work-up are crucial in order to differentiate the underlying disorder. Unfortunately due to some confounding factors, the exact diagnosis cannot be performed in some cases. Moreover, differential identification of exact cause of thyrotoxicosis is also important for medical therapy and follow-up visits for subsequent observations, because GD and TA require further therapy with antithyroid medications and likely requires thyroid operation and SAT is a self-limited inflammation related disorder in which the usual therapy is anti-inflammatory drugs $(4,5)$.

Thyroid hormones are crucial for the normal development, differentiation, proliferation, metabolic balance and physiological function of virtually all tissues (6). The functions of THs are also exceedingly pleiotropic, influencing a number of body tissues at diverse developmental stages. By affecting and regulating haematopoiesis in the bone marrow, it has been reported that thyroid dysfunction can cause alterations in haematological parameters, such as haematocrit (Hct), haemoglobin (Hgb), mean platelet volume (MPV), white 
blood cell $(\mathrm{WBC})$ and its differentials $(7,8)$. Enlarging literature data suggests that hyperthyroidism is related with increased risks for leucopenia, neutropenia, thrombocytopaenia, and WBC differentials including altered platelet-to-lymphocyte ratio (PLR) and neutrophil-to-lymphocyte ratio (NLR) $(1,9)$.

Mean platelet volume (MPV), NLR, and PLR are simple and quick to compute hematologic markers that have been demonstrated to suggest inflammatory response and disease activity in several disease conditions comprising ulcerative colitis, acute pancreatitis, acute appendicitis, gestational diabetes mellitus, cirrhosis, coronary artery disease, neoplastic disorders (10-14). Moreover, there are several reports depicting the importance of NLR, PLR and MPV in differentiating the central causes of thyrotoxicosis (7-9). Unfortunately, these findings were inconsistent across studies, many of which were restricted by relatively undersized sample range and by the lack of replication. Thus, there are still no clinically defined diagnostic characteristics with respect to complete blood cell counts $(\mathrm{CBC})$ which can discriminate the etiology of thyrotoxicosis. In this manner, the present research aimed to analyze the diagnostic significance of NLR, PLR and MPV in thyrotoxicosis patients with distinct etiologies.

\section{MATERIAL AND METHOD}

The study was approved by the institutional ethics committee of COMU (decision no:14-22, dated 09.12.2020). All procedures were performed adhered to the ethical rules and the Helsinki Declaration of Principles.

A total of 46 patients with GD, 39 patients with TA, 46 patients with TMNG and 45 patients with SAT admitted to the endocrinology clinic of Çanakkale Onsekiz Mart University (COMU) Training and Research Hospital between April 2016 and November 2020 included in the present study. The healthy controls were recruited from healthy persons without any history of acute or chronic inflammatory diseases admitted to internal medicine department for routine check-up.

Graves disease diagnosis was established on the existence of regular symptoms of hyperthyroidism and laboratory criteria, including elevated serum TH levels, decreased thyroid-stimulating hormone (TSH) values, positive TSH receptor antibody (TRAb), and/or high radioactive iodine (RAI) uptake tests. Toxic adenoma/multinodular goiter was diagnosed by the use of RAI uptake with/ without thyroid scan and fine-needle aspiration. Diagnosis of SAT was made on the basis of conventional clinical indicators, elevated erythrocyte sedimentation rate (ESR), and pronounced decrease of thyroid RAI uptake.
Exclusion criterias included patients under 18 years old, pregnant women, patients having hematologic or neoplastic disease conditions, serious renal or hepatic disease, chronic inflammatory or autoimmune disorders, use of antithyroid drugs at the time of disease diagnosis. Those having chronic rheumatologic conditions, active or chronic infectious disease, diabetes mellitus, active thyroid orbitopathy and smokers were also excluded.

After database access was granted by the management of the COMU medical center, demographic data of the patients and biochemical and hormonal parameters including $\mathrm{CBC}$ parameters including $\mathrm{Hgb}, \mathrm{Htc}, \mathrm{WBC}$, neutrophil count, lymphocyte count and MPV at diagnosis were extracted from COMU Hospital Information and Management System (HIMS). NLR and PLR were calculated for each study participant from the differential count by dividing the absolute neutrophil count by the absolute lymphocyte count and by dividing the platelet count by the lymphocyte count respectively. All blood samples were collected without the use of any anticoagulant from the antecubital vein after an overnight fast and all CBC analyses were carried out in the hematology laboratory of COMU medical center.

\section{Statistical Analysis}

Statistical analysis was done by using SPSS 22 (SPSS Inc., Chicago, IL, USA). Data are presented as mean \pm standard deviation (SD) for normally distributed variables, median (minimum-maximum) for non-normally distributed variables, and as the number of cases (\%) for nominal variables. To assess differences between groups, a chisquare test was performed for categorical variables. The comparisons between groups were performed by ANOVA and Kruskal-Wallis for parametric and nonparametric variables respectively. Post hoc comparisons were evaluated with Tukey's test for parametric variables and Bonferroni correction Mann-Whitney $U$ test for nonparametric variables. $\mathrm{P}<0.05$ were considered as significant.

\section{RESULTS}

This study included 176 patients (46 GD; 46 TMNG; 45 SAT; and 39 TA patients) with a diagnosis of thyrotoxicosis and a control group with 45 healthy individuals. NLR, PLR were $176.07 \pm 59.59$ and $112.04 \pm 46.49$ respectively. Mean MPV levels of GD, SAT, TMNG, TA and controls were $9.04 \pm 1.35,8.34 \pm 0.96,8.88 \pm 1.25,8.88 \pm 0.92$ and $9.99 \pm 1.39$ respectively. A statistically significant decrease in MPV levels was observed in patients with thyrotoxicosis $(\mathrm{p}<0.001)$. Table 1 summarizes demographic characteristics and biochemical and hormonal data of study participants and controls. Mean NLR levels of SAT patients and controls were $2.99 \pm 1.47$ and $1.73 \pm 0.66$ respectively. Mean MPV and PLR levels of SAT patients and controls were significantly different between groups. 
Table 1. Demographic and laboratory characteristics of study participants

\begin{tabular}{|c|c|c|c|c|c|c|}
\hline & $\begin{array}{l}\text { TA patients } \\
(\mathbf{n}=39)\end{array}$ & $\begin{array}{l}\text { TMNG patients } \\
\quad(\mathrm{n}=46)\end{array}$ & $\begin{array}{l}\text { GD patients } \\
\quad(n=46)\end{array}$ & $\begin{array}{l}\text { SAT patients } \\
\quad(n=45)\end{array}$ & $\begin{array}{l}\text { Control group } \\
\qquad(\mathrm{n}=45)\end{array}$ & Pvalue $^{\star}$ \\
\hline Age, years & $64.97 \pm 10.14$ & $69.23 \pm 8.33$ & $51.00 \pm 16.37$ & $44.62 \pm 9.86$ & $49.22 \pm 14.49$ & 0.000 \\
\hline Gender (F/M) & $24 / 15$ & $34 / 12$ & $31 / 15$ & $42 / 3$ & $27 / 18$ & 0.003 \\
\hline $\operatorname{Hgb}(\mathrm{g} / \mathrm{dl})$ & $13.64 \pm 1.58$ & $13.62 \pm 1.56$ & $12.91 \pm 1.44$ & $12.01 \pm 1.25$ & $13.46 \pm 1.79$ & 0.000 \\
\hline Plt (x1000/ml) & $241.30 \pm 71.34$ & $242.93 \pm 56.43$ & $247.15 \pm 65.37$ & $341.86 \pm 80.89$ & $260.33 \pm 80.25$ & 0.000 \\
\hline $\mathrm{WBC}(\mathrm{x} 1000 / \mathrm{ml})$ & $7.38 \pm 2.17$ & $7.73 \pm 1.98$ & $6.75 \pm 1.75$ & $8.46 \pm 1.69$ & $7.39 \pm 1.94$ & 0.001 \\
\hline TSH (mIU/L) & $0.09 \pm 0.24$ & $0.12 \pm 0.24$ & $0.03 \pm 0.05$ & $0.07 \pm 0.11$ & $2.20 \pm 0.98$ & 0.000 \\
\hline Free T4 (ng/dL) & $1.43 \pm 0.81$ & $1.23 \pm 0.33$ & $2.31 \pm 1.69$ & $2.33 \pm 0.98$ & - & 0.000 \\
\hline Free T3 (ng/dL) & $5.24 \pm 2.93$ & $4.73 \pm 2.35$ & $7.87 \pm 6.94$ & $5.20 \pm 1.54$ & - & 0.001 \\
\hline Anti-TPO (+/-) & $3 / 36$ & $6 / 40$ & $8 / 38$ & $2 / 43$ & - & 0.204 \\
\hline Anti-Tg (+/-) & $2 / 37$ & $6 / 40$ & $8 / 38$ & $4 / 41$ & - & 0.312 \\
\hline CRP (mg/dl) & $0.64 \pm 0.63$ & $0.62 \pm 0.48$ & $0.60 \pm 0.71$ & $5.04 \pm 4.13$ & $0.49 \pm 0.55$ & 0.000 \\
\hline $\operatorname{ESR}(\mathrm{mm} / \mathrm{h})$ & $19.51 \pm 12.88$ & $20.97 \pm 10.10$ & $18.00 \pm 10.90$ & $61.95 \pm 27.72$ & $15.60 \pm 10.16$ & 0.000 \\
\hline NLR & $2.21 \pm 1.43$ & $2.18 \pm 1.10$ & $2.17 \pm 2.17$ & $2.99 \pm 1.47$ & $1.73 \pm 0.66$ & 0.002 \\
\hline PLR & $118.78 \pm 56.93$ & $114.81 \pm 41.57$ & $131.76 \pm 82.63$ & $176.07 \pm 59.59$ & $112.04 \pm 46.49$ & 0.000 \\
\hline MPV (fl) & $8.88 \pm 0.92$ & $8.88 \pm 1.25$ & $9.04 \pm 1.35$ & $8.34 \pm 0.96$ & $9.99 \pm 1.39$ & 0.000 \\
\hline
\end{tabular}

\section{DISCUSSION}

In post hoc analysis, NLR and PLR were significantly higher in the SAT group when compared to the GD, TA, TMNG and control groups ( $\mathrm{p}<0.005$ for each group). MPV levels were different between groups $(\mathrm{p}=0.000)$ in post hoc analysis. Figure 1 shows the levels of hematologic parameters within each study groups and controls.
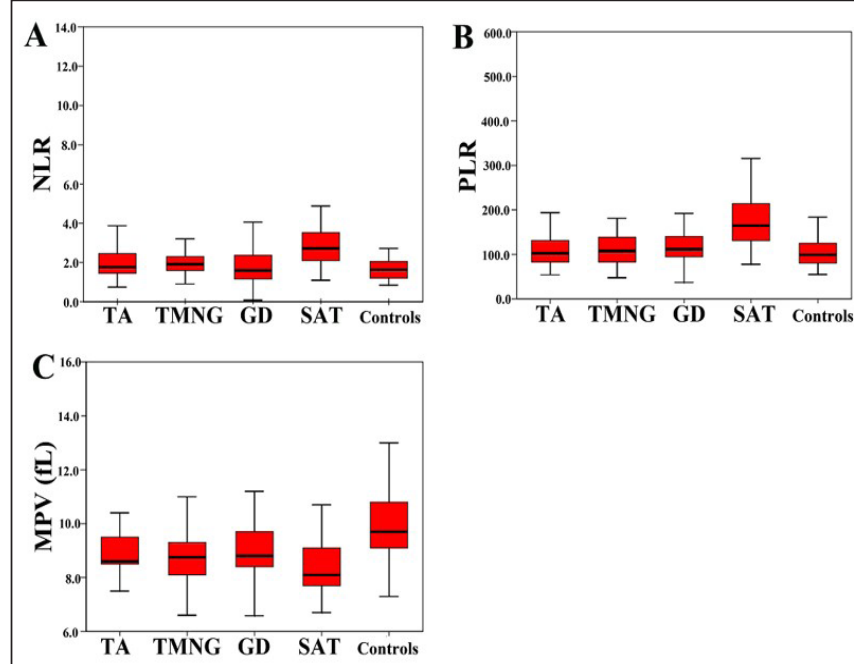

Figure 1. Comparison of NLR (A), PLR (B) and MPV (C) levels within each study group (NLR: Neutrophil-to-lymphocyte ratio, PLR: platelet-to-lymphocyte ratio, MPV: Mean platelet volume, TA: Toxic adenoma, TMNG: Toxic multinodular goiter, GD: Graves' disease, SAT: Subacute thyroiditis).
In this study, we revealed that NLR and PLR is increased in SAT patients and MPV is decreased in all causes of thyrotoxicosis. These findings clearly suggests the role of MPV and WBC differentials in the pathophysiology of thyrotoxicosis particularly in SAT.

Thyroid hormones take part a significant physiological role in the metabolism, growth and development of the human body. The relation between thyroid disorders and abnormalities in haematological parameters by affecting bone marrow is a well-known issue (8). In this context, MPV is one of the determinants of the platelet function which is situated in the routine $\mathrm{CBC}$ analysis and was shown to be affected in distinct disease conditions including acute pancreatitis, inflammatory bowel diseases, cardiac disorders, peritonitis, autoimmune disorders and tumoral diseases $(10-13,15,16)$. The diagnostic role of MPV in thyroid diseases is a novel topic of research with conflicting results. In a study by Simsek et al. (17) the role of MPV in hyperthyroid toxic adenoma patients undergoing radioactive iodine (RAI) ablation treatment was evaluated. It was demonstrated that MPV levels are decreased after radioactive iodine therapy in hyperthyroid patients. In a recent study by Bagir et al. (18) higher initial MPV levels in GD patients and significant drop following the restoration of hyperthyroidism was reported. Lippi et al. (19) reported a considerable relation between MPV and TSH valevels 
in both univariable $(\mathrm{p}<0.001 ; \mathrm{r}=0.12)$ and multivariable logistic regression analysis (beta coefficient, $0.07 ; \mathrm{p}<0.001$ ) after adjustment for multiple confounders including patient age, gender, free T4 values and thrombocyte counts. After homogenization of the study population in context to quartiles of serum TSH levels, a graded increase of MPV values was observed from the first to the fourth quartile of TSH. Contrary to these findings a recent study from China reported no significant relationship between MPV or platelet distribution width (PDW) and THs in a Chinese patient cohort (20). This study demonstrated low MPV levels in all 4 groups of patients with thyrotoxicosis. Low grade inflammation that can be seen in the clinical course of the disease may be responsible for the relation between MPV and thyrotoxicosis. MPV is an initial parameter of platelet activation and big platelets are further reactive than their smaller counterparts in releasing different kinds of pro-inflammatory cytokines that is more likely to aggregate. Therefore, aggregation that is triggered by inflammation causes severe infiltration of large platelets into vascular and intestinal wall, and a final decrease in platelet size (21-23).

In addition to the change of total leukocytic count, alterations of WBC and its differentials, such as NLR and PLR have been proved to be associated with distinct causes of thyrotoxicosis. Moreover, the final outcome of anti-thyroid medical treatment on the granulocytic series are notable and the data on the direct effect of thyroid disorders on granulocytes are partially restricted. Furthermore, literature data on lymphocyte subpopulation distributions in thyroid patients with or without neutropenia are also insufficient (24-25). In this context, SAT which is distinguishable by painful swelling of the thyroid, systemic inflammatory symptoms, and transient thyrotoxicosis is reported to be associated with higher NLR and PLR levels in several studies $(4,26)$. An elegant study by Taşkaldıran et al. (4) the relationship between WBC differentials and the most common causes of thyrotoxicosis including GD, toxic multinodular goiter (TMNG), TA and SAT were investigated. Authors demonstrated higher NLR and PLR levels in the SAT group when compared to other etiologies of thyrotoxicosis. The present study demonstrated elevated NLR and PLR levels in SAT patients compared to the GD, TA, TMNG and healthy controls. Among these, the difference in NLR and PLR was statistically significant. We think that this association might be related to the activation of monocytes and platelets early in the disease process seen in SAT as well as an increase in the number of platelets.

This study didn't observe any significant association between NLR and PLR with other causes of thyrotoxicosis including GD which is the most common reason of hyperthyroidism. GD is an autoimmune disorder, in which the human body produces specific antibodies to the receptor for TSH. Based on this inflammatory background, it is not surprising to find out studies evaluating the role of NLR and PLR in GD. In this context, Taskaldiran et al. (4) investigated the role of these peripheral blood differentials in distinct causes of thyrotoxicosis including GD. Similar to our results authors' demonstrated insignificant association between these parameters and GD. Contrary to our findings Turan et al. (27) revealed higher lymphocyte, monocyte, platelet levels and lower NLR levels in GD patients compared to the healthy controls. Although not compared to healthy population, a study by Alay et al. (28) revealed a statistically significant decrease in NLR in GD compared to TMNG group after treatment.

The main limitation of the present study is the retrospective nature of analysis and relatively low numbers of cases. Moreover, due to the different age distribution of thyrotoxicosis causes, mean ages were different between groups. Another important limitation of our study was the lack of simultaneous measurement of inflammatory markers such as tumor necrosis factor alfa, interleukin 1 beta, interleukin 6 and interleukin 8 with $\mathrm{CBC}$. We accept that it would be noteworthy to demonstrate a possible association between these inflammatory markers and $\mathrm{CBC}$ indices.

\section{CONCLUSION}

This study evaluated the role of NLR, PLR and MPV in newly diagnosed thyrotoxic patients. We strongly believe that NLR and PLR may be helpful to differentiate SAT from other causes of thyrotoxicosis before applying more expensive and difficult diagnostic studies such as RAI uptake.

\section{ETHICAL DECLARATIONS}

Ethics Committee Approval: The study was carried out with the permission of Research Ethics COMU (decision no:14-22, dated: 09.12.2020).

Informed Consent: Because the study was designed retrospectively, no written informed consent form was obtained from patients.

Referee Evaluation Process: Externally peer-reviewed.

Conflict of Interest Statement: The authors have no conflicts of interest to declare.

Financial Disclosure: The authors declared that this study has received no financial support.

Author Contributions: All of the authors declare that they have all participated in the design, execution, and analysis of the paper, and that they have approved the final version. 


\section{REFERENCES}

1. Kim M, Kim BH, Jang MH, et al. High neutrophil-to-lymphocyte ratio is associated with relapse in Graves' disease after antithyroid drug therapy. Endocrine 2020; 67: 406-11.

2. Sharma M, Aronow WS, Patel L, Gandhi K, Desai $H$. Hyperthyroidism. Med Sci Monit 2011; 17: 85-91.

3. Ross DS, Burch HB, Cooper DS, et al. 2016 American Thyroid Association Guidelines for Diagnosis and Management of Hyperthyroidism and Other Causes of Thyrotoxicosis. Thyroid 2016; 26: 1343-421.

4. Taşkaldiran I, Omma T, Önder ÇE, et al. Neutrophil-to-lymphocyte ratio, monocyte-to-lymphocyte ratio, and platelet-tolymphocyte ratio in different etiological causes of thyrotoxicosis. Turk J Med Sci 2019; 49: 1687-92.

5. Fatourechi V, Aniszewski JP, Fatourechi GZ, Atkinson EJ, Jacobsen SJ. Clinical features and outcome of subacute thyroiditis in an incidence cohort: Olmsted County, Minnesota, study. J Clin Endocrinol Metab 2003; 88: 2100-5.

6. Pascual A, Aranda A. Thyroid hormone receptors, cell growth and differentiation. Biochim Biophys Acta 2013; 1830: 3908-16.

7. Franchini M, Montagnana M, Manzato F, Vescovi PP. Thyroid dysfunction and hemostasis: an issue still unresolved. Semin Thromb Hemost 2009; 35: 288-94.

8. Kutluturk F, Gul SS, Sahin S, Tasliyurt T. Comparison of mean platelet volume, platelet count, neutrophil/lymphocyte ratio and platelet/lymphocyte ratio in the euthyroid, overt hypothyroid and subclinical hyperthyroid phases of papillary thyroid carcinoma. Endocr Metab Immune Disord Drug Targets 2019; 19: 859-65.

9. Kawa MP, Grymula K, Paczkowska E, et al. Clinical relevance of thyroid dysfunction in human haematopoiesis: biochemical and molecular studies. Eur J Endocrinol 2010; 162: 295-305.

10. Torun S, Tunc BD, Suvak B, et al. Assessment of neutrophillymphocyte ratio in ulcerative colitis: a promising marker in predicting disease severity. Clin Res Hepatol Gastroenterol 2012; 36: 491-7.

11. Beyazit Y, Sayilir A, Torun S, et al. Mean platelet volume as an indicator of disease severity in patients with acute pancreatitis. Clin Res Hepatol Gastroenterol 2012; 36: 162-8.

12. Suvak B, Torun S, Yildiz H, et al. Mean platelet volume is a useful indicator of systemic inflammation in cirrhotic patients with ascitic fluid infection. Ann Hepatol 2013; 12: 294-300.

13. Yan Q, Ertao Z, Zhimei Z, et al. Systemic immune-inflammation index (SII): A More Promising Inflammation-Based Prognostic Marker for Patients with synchronic colorectal peritoneal carcinomatosis. J Cancer 2020; 11: 5264-72.

14. Butt K, D'Souza J, Yuan C, et al. Correlation of the neutrophil-tolymphocyte ratio (NLR) and platelet-to-lymphocyte ratio (PLR) with contrast-induced nephropathy in patients with acute coronary syndrome undergoing percutaneous coronary interventions. Cureus 2020; 12: e11879.

15. Ulu SM, Asık G, Acay A, et al. Is mean platelet volume a possible marker in acute peritonitis?. Eur J Gen Med 2015; 12: 13-7.

16. Yayla ME, İlgen U, Okatan İE, et al. Association of simple hematological parameters with disease manifestations, activity, and severity in patients with systemic sclerosis. Clin Rheumatol 2020; 39: 77-83.

17. Simsek E, Timur O, Carlioglu A, Durmaz SA, Demirci M, Sevimli $\mathrm{H}$. Mean platelet volume in hyperthyroid toxic adenoma patients after radioactive 131I treatment. Adv Endocrinol 2015; 436768.

18. Bağır GS, Ertörer ME. Higher mean platelet volume is accompanied with hyperthyroidism in patients with Graves' disease. Cukurova Med J 2020; 45: 181-5.

19.Lippi G, Danese E, Montagnana M, Nouvenne A, Meschi T, Borghi L. Mean platelet volume is significantly associated with serum levels of thyroid-stimulating hormone in a cohort of older euthyroid subjects. Endocr Res 2015; 40: 227-30.
20. Ren X, Meng Z, Liu M, et al. No associations exist between mean platelet volume or platelet distribution width and thyroid function in Chinese. Medicine (Baltimore) 2016; 95: e4573.

21. Korniluk A, Koper-Lenkiewicz OM, Kamińska J, Kemona H, Dymicka-Piekarska V. Mean platelet volume (MPV): new perspectives for an old marker in the course and prognosis of inflammatory conditions. Mediators Inflamm 2019; 2019: 9213074.

22. Gasparyan AY, Sandoo A, Stavropoulos-Kalinoglou A, Kitas GD. Mean platelet volume in patients with rheumatoid arthritis: the effect of anti-TNF- $\alpha$ therapy. Rheumatol Int 2010; 30: 1125-9.

23. Wang X, Cui MM, Xu Y, et al. Decreased mean platelet volume predicts poor prognosis in invasive bladder cancer. Oncotarget 2017; 8: 68115-22.

24. Toth EL, Mant MJ, Shivji S, Ginsberg J. Propylthiouracil-induced agranulocytosis: an unusual presentation and a possible mechanism. Am J Med 1988; 85: 725-7.

25. Kyritsi EMA, Yiakoumis X, Pangalis GA, et al. High frequency of thyroid disorders in patients presenting with neutropenia to an outpatient hematology clinic STROBE-compliant article. Medicine (Baltimore) 2015; 94: e886.

26. Cengiz H, Varim C, Demirci T, Cetin S. Hemogram parameters in the patients with subacute thyroiditis. Pak J Med Sci 2020; 36: 2405 .

27.Turan E. Evaluation of neutrophil-to-lymphocyte ratio and hematologic parameters in patients with Graves' disease. Bratisl Lek Listy 2019; 120: 476-80.

28. Alay M, Sönmez GM, Yıldız S. Evaluation of neutrophil-tolymphocyte ratio and mean platelet volume in patients with hyperthyroidism. East J Med 2020; 25: 388-92. 